\title{
Regeneração natural de cerrado sob plantio de Eucalyptus camaldulensis Dehn. no norte de Minas Gerais, Brasil ${ }^{1}$
}

\author{
Michellia Pereira Soares ${ }^{2}$, Yule Roberta Ferreira Nunes ${ }^{3}$
}

\section{RESUMO}

A formação de paisagens homogêneas tornou-se um problema na biologia da conservação, já que essas plantações interferem diretamente na dinâmica das comunidades. Sendo assim, o objetivo deste trabalho foi determinar a composição florística e a estrutura da regeneração natural de cerrado, no sub-bosque de um plantio de Eucalyptus camaldulensis, e verificar se essa regeneração se diferencia, dependendo da distância em relação às bordas do talhão. O estudo foi realizado no município de Montes Claros, MG. Para o levantamento, foram demarcados dois blocos, cada um subdividido em nove parcelas de $10 \mathrm{~m}$ x $10 \mathrm{~m}$, utilizando-se, como critério de inclusão, a altura $\geq 0,30 \mathrm{~m}$. Foram registrados 2.413 indivíduos. No bloco 1, foram amostrados 914 indivíduos, pertencentes a 63 espécies e 26 famílias, sendo Eugenia dysenterica, Hymenaea stigonogocarpa, Calliandra sp. e Duguetia furfuracea as espécies mais importantes, segundo o Valor de Importância (VI). No bloco 2, foram registrados 1.499 indivíduos, distribuídos em 71 espécies e 30 famílias, sendo os maiores VIs alcançados por Cupania vernalis, Chamaecrista desvauxii, Pouteria ramiflora e Senna rugosa. A distribuição em classes de altura e de diâmetro mostrou a maioria dos indivíduos nas menores classes, demonstrando que a área ainda reflete um evento de regeneração recente. Entretanto, os resultados deste estudo indicam que existem comunidades distintas no local, estando o bloco 2 em estádio mais avançado de regeneração do que o primeiro. Esse fato foi confirmado pela análise de agrupamento entre as parcelas e as diferenças de diversidade e desenvolvimento estrutural da vegetação de cada bloco.

Palavras-chave: fitossociologia, composição florística, cerrado.

\section{ABSTRACT}

\section{Natural regeneration of cerrado under plantation of Eucalyptus camaldulensis Dehn. in the north of Minas Gerais, Brazil}

The formation of homogeneous landscapes has become a problem in conservation biology, since these crops directly interfere in the dynamics of communities. Therefore, the objective of this study was to determine the floristic composition and structure of the natural regeneration of cerrado in the understory of Eucalyptus camaldulensis and verify whether the regeneration differs depending on the distance from the edges of the stand. The present work was carried out in the municipality of Montes Claros, MG. Two plots were demarcated for the survey, each subdivided into nine subplots of 10x10 m each, using the height e" $0.30 \mathrm{~m}$ as the inclusion criterion. A total of 2,413 individuals were recorded. In Plot 1, we sampled 914 individuals belonging to 63 species and 26 families; Eugenia dysenterica, Hymenaea stigonogocarpa, Calliandra sp. and Duguetia furfuracea were the most important species according to the Importance

Recebido para publicação em 10/10/2011 e aprovado em 04/02/2013.

${ }^{1}$ Parte da monografia de conclusão de curso de graduação da primeira autora.

${ }^{2}$ Bióloga, Doutora. Instituto Federal de Educação, Ciência e Tecnologia Goiano, Campus Rio Verde, Rodovia Sul Goiana, Km 01, Zona Rural, 75901-970, Rio Verde, Goiás, Brasil. michelliabot@gmail.com (autora para correspondência).

${ }^{3}$ Bióloga, Doutora. Departamento de Biologia Geral, Universidade Estadual de Montes Claros, Avenida Doutor Ruy Braga, s/n, Vila Mauricéia, 39401-089, Montes Claros, Minas Gerais, Brasil. yule.rfnunes@gmail.com 
Value (IV). In Plot 2, 1,499 individuals were distributed in 30 families and 71 species, and the highest IVs were achieved by Cupania vernalis, Chamaecrista desvauxii, Pouteria ramiflora and Senna rugosa. The distribution of classes in height and diameter showed the majority of individuals in the lower classes demonstrating that area still reflect recent event regeneration. However, the results of this study indicate that there are distinct communities at the site and that Plot 2 is in a more advanced stage of regeneration than the Plot 1 . This fact was confirmed by cluster analysis between plots and the differences in diversity and structural development of the vegetation in each plot.

Key words: phytosociological survey, floristic composition, cerrado.

\section{INTRODUÇÃO}

Durante a década de 70, a região norte do Estado de Minas Gerais se notabilizou pela ocupação desordenada do cerrado local, por meio da expansão da monocultura de eucalipto. Esse processo foi subsidiado pelo Governo, a fim de acelerar o crescimento econômico da região e de diminuir a miséria e a pobreza existentes. Em adição, houve a necessidade de atender a demanda por carvão vegetal para o polo siderúrgico e por madeira, para as indústrias de papel e celulose (Brasil, 1984).

Já no fim do século passado, em todo o Estado, a exploração madeireira com finalidades industriais passou de uma origem natural, exploratória, para quase exclusivamente o plantio de florestas homogêneas. Essas florestas tornaram-se, de fato, necessárias, quando a disponibilidade dos recursos naturais estava na contramão de uma premissa sustentável, fator que embasou a legislação ambiental em vigor. Assim, as espécies exóticas arbóreas de rápido crescimento, sobretudo os gêneros Pinus e Eucalyptus, conquistaram grande parte do mercado madeireiro crescente (Vale, 2004). Entretanto, a formação de paisagens homogêneas tornou-se um problema na biologia da conservação, já que estas plantações interferem diretamente na dinâmica das comunidades, uma vez que aumentam a fragmentação de habitats naturais e, consequentemente, elevam as taxas de extinção e isolamento reprodutivo dessas populações (Metzger \& Décamps, 1997). As alterações nas comunidades de fauna e flora locais perturbam o equilíbrio alcançado pelas populações ao longo do tempo, por meio de seus mecanismos de autorregulação, e os novos padrões e condições de sombreamento, competição (por água e nutrientes) e possíveis efeitos alelopáticos resultam em uma estruturação populacional erodida geneticamente e de diversidade alfa baixa (Almeida, 1987; Silva, 1994). Mesmo assim, em muitos desses plantios, dependendo do tipo de manejo aplicado no talhão, observa-se a presença de um subbosque formado a partir da regeneração natural de es- pécies nativas (Aubert \& Oliveira-Filho, 1994; Neri et al., 2005; Alencar et al., 2011; Venzke et al., 2012). Essa vegetação secundária surge após o abandono das áreas (Rodon Neto, 1999) e, no Domínio do Cerrado, cuja resiliência é maior, comparada com a dos outros domínios (Metzger, 2003), este evento de regeneração é ainda mais comum, resultando em eficiente medida de restauração.

A necessidade de se conhecer a composição e a estrutura da vegetação natural, em condições de perturbação e regeneração subsequente, é ponto primordial para as decisões práticas que têm por objetivo a restauração ecológica do ecossistema (Sartori, 2001). Apesar disto, poucos estudos foram feitos, abordando a sucessão secundária em áreas perturbadas pela ação antrópica (Aubert \& Oliveira-Filho, 1994), principalmente com plantios homogêneos de espécies exóticas (Aubert \& Oliveira-Filho 1994; Silva, 1994; Nappo et al.; 1999; Nappo et al., 2000; Sartori, 2001; Neri et al., 2005; Viani et al., 2010; Alencar et al., 2011; Venzke et al., 2012), sendo a maioria no Domínio Attântico e poucos no Domínio Cerrado. Por essa razão, este trabalho teve como objetivo determinar a composição florística, nas unidades amostrais (parcelas), e a estrutura fitossociológica da regeneração natural de Cerrado, presente no sub-bosque de um plantio homogêneo de Eucalyptus camaldulensis Dehn., e verificar se essa regeneração apresenta diferenças florísticas e estruturais, dependendo da distância em relação às bordas do talhão.

\section{MATERIAL E MÉTODOS}

\section{Área de estudo}

Este trabalho foi realizado na Fazenda Três Irmãos, propriedade da Companhia Paulista de Ferro e Liga, no município de Montes Claros, norte de Minas Gerais. O clima da região é do tipo semiárido, com duas estações bem definidas, sendo uma chuvosa (de outubro a março) e uma estação seca (de abril a setembro). A temperatura média oscila em torno $23^{\circ} \mathrm{C}$ e, a precipitação anual, ao redor de $1.000 \mathrm{~mm} / \mathrm{ano}$, com chuvas concentradas nos 
meses de novembro a janeiro. O relevo caracteriza-se por áreas aplainadas, superfícies onduladas e pedimentos ravinados. Os solos predominantes são os Latossolos Vermelho-Escuro eutróficos, os Podzólicos VermelhoAmarelo eutróficos e Cambissolos Álicos e distróficos (Embrapa et al., 1976). Fisionomicamente, a região encontra-se na transição dos Domínios do Cerrado e da Caatinga, apresentando como principais fisionomias a Mata Seca (Floresta Estacional Decidual), que é predominante na área, e o Cerrado sentido restrito (Nunes et al., 2005).

A vegetação original da área estudada era constituída por um continuum de Cerrado sentido restrito, que foi fragmentada por uma estrada de acesso a povoados próximos. Atualmente, de um lado da estrada está um remanescente de Cerrado e, do outro, o plantio de Eucalyptus camaldulensis. Entre os anos de 1988 e 1989, a cobertura vegetal nativa foi substituída pelo plantio homogêneo, com espaçamento de $3 \times 2 \mathrm{~m}$, em uma área de aproximadamente 333 ha. A extração parcial da madeira foi realizada apenas uma vez, no ano de 1997. Após essa retirada, sem destoca e reforma de talhão, não houve qualquer outra intervenção no local, ou seja, a área foi abandonada e deixada à ação do tempo. A partir de processos naturais de regeneração, formou-se um sub-bosque de espécies nativas.

\section{Caracterização da vegetação}

Para o levantamento da vegetação regenerante, foram demarcados dois blocos de amostragem na área, com nove parcelas de $10 \times 10 \mathrm{~m}$ cada um, subdivididas em três transectos contíguos. O primeiro bloco foi alocado na borda do plantio, a $30 \mathrm{~m}$ da estrada de acesso e a $100 \mathrm{~m}$ de uma área de Cerrado preservado. O segundo bloco foi demarcado no interior da floresta, a cerca de $200 \mathrm{~m}$ de distância do primeiro e orientado no mesmo sentido. $\mathrm{Na}$ amostragem, foram incluídos todos os indivíduos com altura $\geq 0,30 \mathrm{~m}$, sendo tomadas medidas de altura e de diâmetro à altura do solo (DAS). Todos os indivíduos amostrados foram marcados com plaquetas de alumínio numeradas.

O material botânico coletado foi identificado por meio de morfologia comparada, consultas a herbários e especialistas. O sistema de classificação adotado para a elaboração da lista florística seguiu o proposto por Angiosperm Phylogeny Group (APG III, 2009).

\section{Análise de dados}

Para a análise da estrutura da comunidade, foram estimados os parâmetros fitossociológicos de densidade, dominância e frequência relativas, além do valor de importância (VI) (Mueller-Dombois \& Ellenberg, 1974). Também foi calculado o índice de diversidade de Shannon (H') (Brower \& Zar, 1984) e a equabilidade (Pielou, 1975), sendo todos os cálculos obtidos por meio do programa
Fitopac 1 (Shepherd, 1996). Além disso, os indivíduos amostrados foram distribuídos em classes de altura e de diâmetro, com amplitude entre as classes de 0,2 m para altura e $1 \mathrm{~cm}$ para os diâmetros.

A existência de similaridade florística entre as parcelas nos diferentes blocos foi verificada por meio de análise de agrupamento, utilizando-se o índice de similaridade de Sorensen (Brower \& Zar, 1984) e o Diagrama de Venn. A interpretação das relações florísticas entre as parcelas foi feita pelo método de agrupamentos pelas médias não ponderadas (UPGMA) (Sneath \& Sokal, 1973). O resultado foi expresso na forma de dendrograma gerado a partir do programa Fitopac 1 (Shepherd, 1996). Essa expressão foi a que melhor representou as similaridades da composição florística entre os dois blocos, justificando sua escolha.

\section{RESULTADOS E DISCUSSÃO}

No sub-bosque de Eucalyptus camaldulensis foram registrados 2.413 indivíduos, distribuídos em 93 espécies. Não foram completamente identificadas 35 espécies (ou $37,63 \%$ da riqueza total), por apresentarem poucas folhas no ato de coleta ou por ainda estarem em estádio juvenil de desenvolvimento, comprometendo suas determinações. No bloco 1, foram amostrados 914 indivíduos, distribuídos em 63 espécies, quatro identificadas apenas como morfoespécies, pertencentes a 26 famílias botânicas. As famílias que apresentaram os maiores números de espécies foram: Myrtacae (7), Leguminosae-Faboideae (7) e Leguminosae-Caesalpinoideae (6) (Tabela 1). O bloco 2 superou o primeiro, tanto com relação à riqueza florística, quanto à abundância de indivíduos. Foram registrados 1.499 indivíduos e um total de 71 espécies, três delas identificadas como morfoespécies, distribuídas em 30 famílias. As famílias Myrtaceae (9), Leguminosae-Faboideae (7), Leguminosae-Caesalpinoideae (6) também apresentaram riqueza elevada, sendo possível destacar Malpighiaceae (6), neste bloco, pela mesma razão (Tabela 2).

O número de espécies exclusivas de cada bloco foi 22 para o bloco 1 e 30 para o bloco 2 (Figura 1), contra 41 espécies comuns aos dois blocos, demonstrando grande similaridade entre borda e interior, mas não uma homogeneidade florística em toda área em regeneração. Com relação à similaridade florística existente entre as parcelas, verificase, pela análise de agrupamento (Figura 2), que se formaram dois grupos bem distintos, sendo que cada grupo corresponde a um bloco de amostragem. As parcelas do bloco 1 foram ligadas a $50 \%$ de similaridade, enquanto o bloco 2 apresentou maior relação florística entre as suas parcelas, que se ligaram a $65 \%$ de similaridade. Esses resultados vêm constatar a formação de comunidades distintas no sub-bosque de Eucalyptus camaldulensis. 
Tabela 1. Parâmetros fitossociológicos em ordem decrescente do valor de importância (VI), das espécies amostradas na regeneração natural sob Eucalyptus camaldulensis, no bloco 1, na Fazenda Três Irmãos, Montes Claros, MG. DR = densidade relativa, FR= frequência relativa, e $\mathrm{DoR}=$ dominância relativa

\begin{tabular}{|c|c|c|c|c|c|}
\hline Espécies & Famílias & DR & FR & DoR & $\% \mathrm{VI}$ \\
\hline Eugenia dysenterica & Myrtaceae & 12,04 & 4,19 & 18,31 & 11,54 \\
\hline Hymenaea stigonocarpa & Leg. Caesalpinoideae & 2,74 & 3,72 & 13,56 & 6,69 \\
\hline Calliandra sp. & Leg. Mimosoideae & 10,72 & 4,19 & 2,50 & 5,82 \\
\hline Duguetia furfuracea & Annonaceae & 8,75 & 4,19 & 4,30 & 5,76 \\
\hline Caryocar brasiliense & Caryocaraceae & 2,08 & 2,33 & 12,51 & 5,65 \\
\hline Cupania vernalis & Sapindaceae & 9,74 & 3,72 & 3,13 & 5,55 \\
\hline Qualea multiflora & Vochysiaceae & 3,39 & 2,79 & 7,51 & 4,58 \\
\hline Eugenia sp. & Myrtaceae & 4,38 & 3,26 & 5,15 & 4,27 \\
\hline Machaerium opacum & Leg. Papilionoideae & 4,27 & 2,79 & 5,06 & 4,05 \\
\hline Campomanesia velutina & Myrtaceae & 6,35 & 4,19 & 1,28 & 3,95 \\
\hline Erythroxylum suberosum & Erythroxylaceae & 1,42 & 2,79 & 2,77 & 2,34 \\
\hline Peritassa campestris & Celastraceae & 3,72 & 1,40 & 1,45 & 2,20 \\
\hline Crotalaria spectabilis & Leg. Papilionoideae & 1,86 & 3,72 & 0,93 & 2,18 \\
\hline Oxalis goyazensis & Oxalidaceae & 2,52 & 3,72 & 0,23 & 2,16 \\
\hline Eriotheca sp. & Malvaceae & 1,86 & 2,79 & 1,60 & 2,09 \\
\hline Dalbergia myscolobium & Leg. Papilionoideae & 1,42 & 2,33 & 2,45 & 2,07 \\
\hline Qualea grandiflora & Vochysiaceae & 0,88 & 0,93 & 3,93 & 1,92 \\
\hline Swartzia sp. & Leg. Papilionoideae & 2,08 & 2,79 & 0,67 & 1,85 \\
\hline Maprounea guianensis & Euphorbiaceae & 2,63 & 1,86 & 0,71 & 1,74 \\
\hline Jacaranda sp. & Bignoniaceae & 1,86 & 2,33 & 0,42 & 1,54 \\
\hline Antonia ovata & Loganiaceae & 0,55 & 1,86 & 1,53 & 1,31 \\
\hline Bauhinia sp. & Leg. Caesalpinoideae & 1,09 & 1,86 & 0,93 & 1,30 \\
\hline Aspidosperma sp. & Apocynaceae & 1,31 & 1,40 & 0,82 & 1,18 \\
\hline Acosmium dasycarpum & Leg. Papilionoideae & 0,66 & 1,40 & 1,34 & 1,13 \\
\hline Pouteria ramiflora & Sapotaceae & 0,44 & 1,40 & 1,53 & 1,12 \\
\hline Myrtaceae sp1 & Myrtaceae & 0,77 & 1,86 & 0,49 & 1,04 \\
\hline Ouratea castaneifolia & Ochnaceae & 0,44 & 1,86 & 0,57 & 0,96 \\
\hline Casearia sylvestris & Salicaceae & 1,09 & 1,40 & 0,27 & 0,92 \\
\hline Aspidosperma tomentosum & Apocynaceae & 0,44 & 1,86 & 0,45 & 0,92 \\
\hline Myrcia eriopus & Myrtaceae & 0,55 & 1,86 & 0,32 & 0,91 \\
\hline Senna chrysocarpa & Leg. Caesalpinoideae & 0,66 & 1,86 & 0,17 & 0,90 \\
\hline Palicourea rigida & Rubiaceae & 0,77 & 1,40 & 0,29 & 0,82 \\
\hline Annona coriacea & Annonaceae & 0,44 & 1,40 & 0,56 & 0,80 \\
\hline Croton sp. & Euphorbiaceae & 0,88 & 1,40 & 0,04 & 0,77 \\
\hline Erythroxylum deciduum & Erythroxylaceae & 0,33 & 1,40 & 0,29 & 0,67 \\
\hline Erythroxylum sp. & Erythroxylaceae & 0,44 & 1,40 & 0,15 & 0,67 \\
\hline Anemopaegma arvense & Bignoniaceae & 0,33 & 0,93 & 0,03 & 0,43 \\
\hline Passiflora sp. & Passifloraceae & 0,33 & 0,93 & 0,02 & 0,43 \\
\hline Peltae sp. & Malvaceae & 0,22 & 0,93 & 0,02 & 0,39 \\
\hline Zornia latifolia & Leg. Papilionoideae & 0,22 & 0,93 & 0,02 & 0,39 \\
\hline Heteropterys byrsonimifolia & Malpighiaceae & 0,11 & 0,47 & 0,36 & 0,31 \\
\hline Byrsonima verbascifolia & Malpighiaceae & 0,33 & 0,47 & 0,12 & 0,31 \\
\hline Indeterminada sp3 & Desconhecida & 0,33 & 0,47 & 0,02 & 0,27 \\
\hline Kielmeyera sp. & Calophyllaceae & 0,11 & 0,47 & 0,20 & 0,26 \\
\hline Dimorphandra mollis & Leg. Caesalpinoideae & 0,11 & 0,47 & 0,20 & 0,26 \\
\hline Indeterminada sp4 & Desconhecida & 0,22 & 0,47 & 0,07 & 0,25 \\
\hline Bauhinia longifolia & Leg. Caesalpinoideae & 0,11 & 0,47 & 0,17 & 0,25 \\
\hline Zeyheria sp. & Bignoniaceae & 0,22 & 0,47 & 0,05 & 0,25 \\
\hline Stryphnodendron adstringens & Leg. Mimosoideae & 0,22 & 0,03 & 0,22 & 0,16 \\
\hline Camptosema sp. & Leg. Papilionoideae & 0,22 & 0,02 & 0,22 & 0,16 \\
\hline Qualea parviflora & Vochysiaceae & 0,11 & 0,12 & 0,11 & 0,16 \\
\hline Casearia arborea & Salicaceae & 0,11 & 0,07 & 0,11 & 0,16 \\
\hline Vernonia sp. & Asteraceae & 0,11 & 0,47 & 0,05 & 0,21 \\
\hline
\end{tabular}

Rev. Ceres, Viçosa, v. 60, n.2, p. 205-214, mar/abr, 2013 
Continuação da Tabela 1

\begin{tabular}{lllccc}
\hline Espécies & Famílias & DR & FR & DoR & \%VI \\
\hline Myrcia variabilis & Myrtaceae & 0,11 & 0,47 & 0,05 & 0,21 \\
Indeterminada sp1 & Desconhecida & 0,11 & 0,47 & 0,05 & 0,21 \\
Indeterminada sp2 & Desconhecida & 0,11 & 0,47 & 0,02 & 0,20 \\
Eucalyptus camaldulensis & Myrtaceae & 0,11 & 0,47 & 0,01 & 0,20 \\
Mimosa sp. & Leg. Mimosoideae & 0,11 & 0,47 & 0,01 & 0,20 \\
Annona sp. & Annonaceae & 0,11 & 0,47 & 0,01 & 0,20 \\
Rubiaceae sp1 & Rubiaceae & 0,11 & 0,47 & 0,01 & 0,19 \\
Bauhinia pulchella & Leg. Caesalpinoideae & 0,11 & 0,47 & 0,01 & 0,19 \\
Mandevilla velutina & Apocynaceae & 0,11 & 0,47 & 0,01 & 0,19 \\
Davilla rugosa & Dilleniaceae & 0,11 & 0,47 & 0,01 & 0,19 \\
\hline
\end{tabular}

Os valores de diversidade florística (H') e equabilidade (J) foram elevados, principalmente no bloco 2 . Os valores de 3,26 e 0,79 , para a diversidade e equabilidade, para o bloco 1, e de 3,49 e 0,82, para o bloco 2, são altos, quando comparados com os de outros trabalhos de regeneração natural, como o de Calegário (1993), que encontrou uma diversidade variando de 3,083 a 3,350, para cinco áreas distintas; o de Duringan et al. (1997), que, trabalhando também em sub-bosque de eucalipto, obteve o valor de 2,138 para diversidade; Saporetti Jr et al. (2003), que encontraram o valor de 2,636 e Medeiros et al. (2007), que obtiveram valor de 3,21 .

Viani et al. (2010), analisando diversos trabalhos publicados sobre o tema de regeneração natural sob plantações florestais, principalmente no Brasil, constataram que esses plantios podem funcionar como redutos da biodiversidade e, também, como facilitadores da restauração ecológica de florestas nativas. A comprovação disto pode ser observada pelo trabalho desenvolvido por Durigan et al. (1997), que compararam a diversidade e riqueza da regeneração natural em plantio de Eucalyptus citriodora com um remanescente de cerradão e verificaram que os valores foram muito próximos. Por outro lado, Neri et al. (2005) e Saporetti Jr et al. (2003) constataram uma menor diversidade no sub-bosque regenerante estudado por eles, quando comparada à de outros remanescentes. Entretanto, a composição florística ainda se assemelhava à da vegetação original. Todas as famílias e espécies que se destacaram neste estudo são características do cerrado, demonstrando que a área está se regenerando com elementos florísticos peculiares da fisionomia.

Quanto à estrutura fitossociológica, no bloco 1 , as oito famílias com os maiores valores de importância (VI) foram: Myrtaceae, Leguminosae-Faboideae, LeguminosaeCaesalpinoideae, Annonaceae, Vochysiaceae, Leguminosae-Mimosoideae, Sapindaceae e Caryocaraceae, representando 69,09\% do total. No segundo bloco, as famílias Leguminosae-Caesalpinoideae, Sapindaceae, LeguminosaeFaboideae, Rubiaceae, Myrtaceae, Vochysiaceae, Leguminosae-Mimosoideae e Sapotaceae totalizaram 70,67\%.
No mesmo sentido, as espécies mais importantes no aspecto da estrutura da comunidade, diferiram entre os blocos de amostragem. As espécies Eugenia dysenterica, Hymenaea stigonocarpa, Calliandra sp., Duguetia furfuracea, Caryocar brasiliense, Cupania vernalis e Qualea multiflora foram as de maior VI no bloco 1 (Tabela 1). O bloco 2 apresenta uma escala diferenciada de VI (Tabela 2), sendo que Cupania vernalis, Chamaecrista desvauxii, Pouteria ramiflora, Senna rugosa, Hymenaea stigonocarpa, Richardia sp. e Calliandra sp. passam a ser as espécies mais importantes.

Diante destes resultados, percebe-se que, apesar da homogeneidade imposta pelo reflorestamento, os diferentes tratos silviculturais pretéritos dessa área, bem como as medidas de manejo e transporte dentro do talhão, podem ocasionar diferenças na vegetação em regeneração. A localização borda-interior também pode ser um fator determinante nessa heterogeneidade do sub-bosque. As bordas são caracterizadas pela modificação abrupta dos fluxos biológicos entre ambientes (Paciência \& Prado, 2004). A formação de borda em uma área pode propiciar diversos efeitos abióticos, como aumento dos ventos, variação na temperatura, penetração vertical da luz (maior radiação solar) e baixa umidade (Davies-colley et al., 2000; Larcher, 2000, Redding et al.,2003). Viani et al. (2010) verificaram também que a densidade de copas e a disponibili-

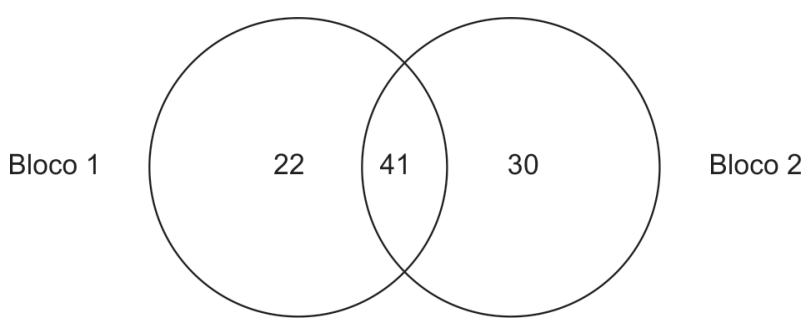

Figura 1. Diagrama de Venn, obtido pelo número de espécies exclusivas de cada bloco e o número de espécies em comum, representado pela intersecção entre os blocos no diagrama. Como base foram utilizados os valores absolutos de riqueza das parcelas amostradas na regeneração natural sob Eucalyptus camaldulensis na Fazenda Três Irmãos, Montes Claros, MG. 
Tabela 2. Parâmetros fitossociológicos por ordem decrescente do valor de importância (VI), das espécies amostradas na regeneração natural sob Eucalyptus camaldulensis, no bloco 2, na Fazenda Três Irmãos, Montes Claros, MG. DR = densidade relativa, FR = frequência relativa e $\mathrm{DoR}=$ dominância relativa

\begin{tabular}{|c|c|c|c|c|c|}
\hline Espécies & Famílias & DR & FR & DoR & $\% \mathrm{VI}$ \\
\hline Cupania vernalis & Sapindaceae & 12,21 & 11,19 & 2,87 & 8,76 \\
\hline Chamaecrista desvauxii & Leg. Caesalpinoideae & 9,14 & 6,49 & 2,87 & 6,17 \\
\hline Pouteria ramiflora & Sapotaceae & 4,00 & 7,68 & 2,87 & 4,85 \\
\hline Senna rugosa & Leg. Caesalpinoideae & 4,74 & 5,68 & 2,55 & 4,32 \\
\hline Hymenaea stigonocarpa & Leg. Caesalpinoideae & 1,93 & 8,01 & 1,91 & 3,95 \\
\hline Richardia sp. & Rubiaceae & 7,20 & 2,08 & 2,55 & 3,94 \\
\hline Calliandra sp. & Leg. Mimosoideae & 7,27 & 1,60 & 2,87 & 3,91 \\
\hline Acosmium dasycarpum & Leg. Papilionoideae & 4,34 & 3,91 & 2,87 & 3,71 \\
\hline Swartzia sp. & Leg. Papilionoideae & 2,47 & 3,62 & 2,87 & 2,98 \\
\hline Myrcia eriopus & Myrtaceae & 2,87 & 3,49 & 2,55 & 2,97 \\
\hline Qualea grandiflora & Vochysiaceae & 1,67 & 5,25 & 1,27 & 2,73 \\
\hline Palicourea rigida & Rubiaceae & 3,27 & 2,31 & 2,23 & 2,60 \\
\hline Copaifera langsdorffii & Leg. Caesalpinoideae & 2,33 & 1,92 & 2,87 & 2,37 \\
\hline Crotalaria spectabilis & Leg. Papilionoideae & 3,07 & 1,52 & 2,23 & 2,27 \\
\hline Duguetia furfuracea & Annonaceae & 1,93 & 1,98 & 2,87 & 2,26 \\
\hline Myrtaceae sp1 & Myrtaceae & 3,20 & 0,91 & 2,55 & 2,22 \\
\hline Qualea multiflora & Vochysiaceae & 0,40 & 4,78 & 1,27 & 2,15 \\
\hline Alibertia sessilis & Rubiaceae & 1,00 & 3,19 & 2,23 & 2,14 \\
\hline Myrcia variabilis & Myrtaceae & 2,07 & 0,91 & 2,87 & 1,95 \\
\hline Senna chrysocarpa & Leg. Caesalpinoideae & 2,20 & 0,97 & 2,55 & 1,91 \\
\hline Maprounea guianensis & Euphorbiaceae & 1,13 & 1,28 & 2,55 & 1,65 \\
\hline Jacaranda sp. & Bignoniaceae & 2,33 & 0,68 & 1,91 & 1,64 \\
\hline Callisthene major & Vochysiaceae & 1,27 & 0,99 & 2,55 & 1,60 \\
\hline Eugenia dysenterica & Myrtaceae & 1,20 & 1,26 & 2,23 & 1,56 \\
\hline Casearia arborea & Salicaceae & 1,47 & 0,63 & 2,55 & 1,55 \\
\hline Annona crassiflora & Annonaceae & 1,40 & 0,92 & 2,23 & 1,52 \\
\hline Bauhinia sp. & Leg. Caesalpinoideae & 1,13 & 1,76 & 0,96 & 1,28 \\
\hline Bowdichia virgilioides & Leg. Papilionoideae & 0,60 & 1,62 & 1,59 & 1,27 \\
\hline Davilla rugosa & Dilleniaceae & 0,87 & 0,37 & 2,55 & 1,26 \\
\hline Enterolobium gummiferum & Leg. Mimosoideae & 0,07 & 2,98 & 0,32 & 1,12 \\
\hline Casearia sylvestris & Salicaceae & 0,60 & 0,81 & 1,91 & 1,11 \\
\hline Roupala montana & Proteaceae & 1,00 & 0,23 & 1,91 & 1,05 \\
\hline Heteropterys byrsonimifolia & Malpighiaceae & 0,33 & 1,27 & 1,27 & 0,96 \\
\hline Erythroxylum sp. & Erythroxylaceae & 0,93 & 0,63 & 1,27 & 0,95 \\
\hline Camptosema sp. & Leg. Papilionoideae & 0,73 & 0,13 & 1,91 & 0,92 \\
\hline Peritassa campestris & Celastraceae & 0,73 & 0,43 & 1,59 & 0,92 \\
\hline Ouratea spectabilis & Ochnaceae & 1,00 & 0,36 & 1,27 & 0,88 \\
\hline Croton sp. & Euphorbiaceae & 0,87 & 0,17 & 1,27 & 0,77 \\
\hline Eugenia sp. & Myrtaceae & 0,40 & 0,31 & 1,59 & 0,77 \\
\hline Stryphnodendron adstringens & Leg. Mimosoideae & 0,13 & 1,60 & 0,32 & 0,68 \\
\hline Miconia albicans & Melastomataceae & 0,33 & 0,22 & 1,27 & 0,61 \\
\hline Byrsonima crassifolia & Malpighiaceae & 0,27 & 0,18 & 1,27 & 0,57 \\
\hline Annona coriacea & Annonaceae & 0,27 & 0,18 & 0,96 & 0,47 \\
\hline Peltae sp. & Malvaceae & 0,33 & 0,04 & 0,96 & 0,44 \\
\hline Ouratea castaneifolia & Ochnaceae & 0,27 & 0,09 & 0,96 & 0,44 \\
\hline Myrcia sp. & Myrtaceae & 0,13 & 0,68 & 0,32 & 0,38 \\
\hline Caryocar brasiliense & Caryocaraceae & 0,07 & 0,69 & 0,32 & 0,36 \\
\hline Eriotheca sp. & Malvaceae & 0,20 & 0,19 & 0,64 & 0,34 \\
\hline Banisteriopsis sp & Malpighiaceae & 0,13 & 0,16 & 0,64 & 0,31 \\
\hline Byrsonima verbascifolia & Malpighiaceae & 0,13 & 0,13 & 0,64 & 0,30 \\
\hline Zeyheria sp & Bignoniaceae & 0,13 & 0,11 & 0,64 & 0,29 \\
\hline Rubiaceae sp2 & Rubiaceae & 0,13 & 0,10 & 0,64 & 0,29 \\
\hline Aspidosperma tomentosum & Apocynaceae & 0,13 & 0,09 & 0,64 & 0,29 \\
\hline
\end{tabular}

Rev. Ceres, Viçosa, v. 60, n.2, p. 205-214, mar/abr, 2013 
Continuação da Tabela 2

\begin{tabular}{llcccc}
\hline Espécies & Famílias & DR & FR & DoR & \%VI \\
\hline Byrsonima sp. & Malpighiaceae & 0,13 & 0,06 & 0,64 & 0,28 \\
Baccharis sp. & Asteraceae & 0,13 & 0,04 & 0,64 & 0,27 \\
Antonia ovata & Loganiaceae & 0,13 & 0,01 & 0,64 & 0,26 \\
Schefflera macrocarpa & Araliaceae & 0,07 & 0,29 & 0,32 & 0,23 \\
Diospyros hispida & Ebenaceae & 0,07 & 0,24 & 0,32 & 0,21 \\
Campomanesia velutina & Myrtaceae & 0,20 & 0,09 & 0,32 & 0,20 \\
Dalbergia violacea & Leg. Papilionoideae & 0,13 & 0,15 & 0,32 & 0,20 \\
Indeterminada sp 6 & Desconhecida & 0,20 & 0,03 & 0,32 & 0,18 \\
Erythroxylum suberosum & Erythroxylaceae & 0,20 & 0,02 & 0,32 & 0,18 \\
Kielmeyera petiolares & Calophyllaceae & 0,07 & 0,13 & 0,32 & 0,17 \\
Cybianthus sp. & Primulaceae & 0,13 & 0,06 & 0,32 & 0,17 \\
Machaerium opacum & Leg. papilionoideae & 0,07 & 0,05 & 0,32 & 0,14 \\
Guapira graciliflora & Nyctaginaceae & 0,07 & 0,03 & 0,32 & 0,14 \\
Byrsonima coccolobifolia & Malpighiaceae & 0,07 & 0,02 & 0,32 & 0,14 \\
Indeterminada sp 5 & Desconhecida & 0,07 & 0,01 & 0,32 & 0,13 \\
Myrtaceae sp2 & Myrtaceae & 0,07 & 0,01 & 0,32 & 0,13 \\
Myrtaceae sp3 & Myrtaceae & 0,07 & 0,01 & 0,32 & 0,13 \\
Indeterminada sp 7 & Desconhecida & 0,07 & 0,01 & 0,32 & 0,13 \\
\hline
\end{tabular}

dade de luz podem ser reguladores para a densidade e riqueza das espécies nativas regenerantes em plantios de espécies exóticas comerciais. Fatores externos, como o manejo do talhão, também devem ser considerados, visto que, na borda, pelas próprias questões de acesso existe um impacto maior. A condição mais sombreada do interior do talhão e menos impactada foi observada, neste estu- do, pela ocorrência de espécies secundárias e de crescimento à sombra, que foram encontradas, com VI's significativos, no bloco 2, como o caso de Myrcia eriopus, Alibertia sessilis e Maprounea guianensis, além de se considerar que esse bloco foi mais rico e mais denso que o bloco $1 \mathrm{e}$, consequentemente, também mais valorizado, quando se considera o sucesso da regeneração natural.

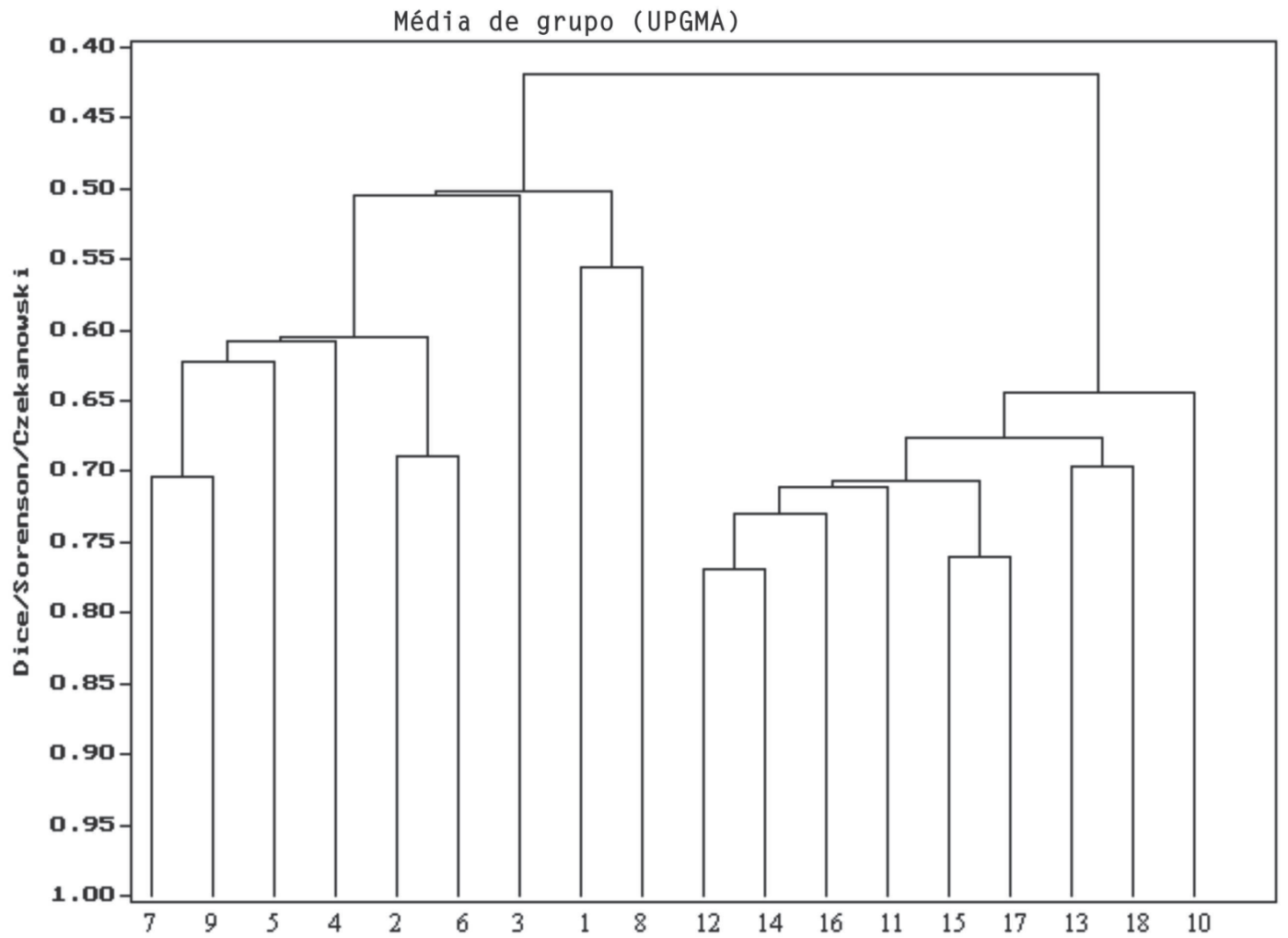

Figura 2. Dendrograma obtido pelo método de médias não ponderadas (UPGMA) com base no índice de Sorensen para as parcelas amostradas na regeneração natural sob Eucalyptus camaldulensis na Fazenda Três Irmãos, Montes Claros, MG. 
Nos dois blocos, a distribuição de altura demonstrou diferenças nos estratos verticais. No bloco 1, a distribuição foi semelhante ao modelo de "J" invertido (Figura 3), proposto por Liocourt (1898) apud Meyer (1952) e, para bloco 2, foi obtido um padrão majoritário intermediário. No primeiro bloco, 94,73\% dos indivíduos ficaram concentrados nas classes mais baixas, ou seja, entre 0,3 e 1,5 m de altura. No bloco 2, essa percentagem foi de $89,51 \%$ para as mesmas classes. A altura máxima atingida no bloco 1 foi de $3,1 \mathrm{~m}$, valor baixo quando comparado com o segundo bloco, que foi de cerca de 5,1 $\mathrm{m}$. Mesmo com uma percentagem de indivíduos pouco representativa nas classes mais altas, o bloco 2 apresenta-se em estádio sucessional mais avançado, haja vista a maior abundância, encontrada nas maiores classes de altura, em comparação com o primeiro bloco, sabendo-se que, na classificação proposta por Ribeiro \& Walter (2008), para o Cerrado Típico, a altura média varia entre três e seis metros.
Para as classes diamétricas, no bloco 1, 84,68\% dos indivíduos foram reunidos nas duas primeiras classes, entre 0,1 e $2 \mathrm{~cm}$ e, no bloco 2, 93,12\% dos indivíduos amostrados pertenciam a esse intervalo (Figura 4). O primeiro bloco apresentou uma melhor distribuição de indivíduos em cada classe, mas semelhantemente à distribuição de alturas, no bloco 2, os indivíduos atingiram valores mais elevados de diâmetros. O valor máximo registrado no bloco 1 foi de $10,80 \mathrm{~cm}$, enquanto, no bloco 2 , foi de $12 \mathrm{~cm}$.

Além da composição florística e análise fitossociológica, os histogramas de altura e diâmetro demonstram que os dois blocos estão em fases sucessionais distintas, por causa das diferenças de abundância encontradas entre as classes. Alguns fatores já mencionados podem estar influenciando neste desenvolvimento, como a localização do bloco 2 no interior do plantio, com uma menor influência da ação antrópica, em decorrência da distância. Entretanto, o bloco 1 está próximo a uma vegetação nati-

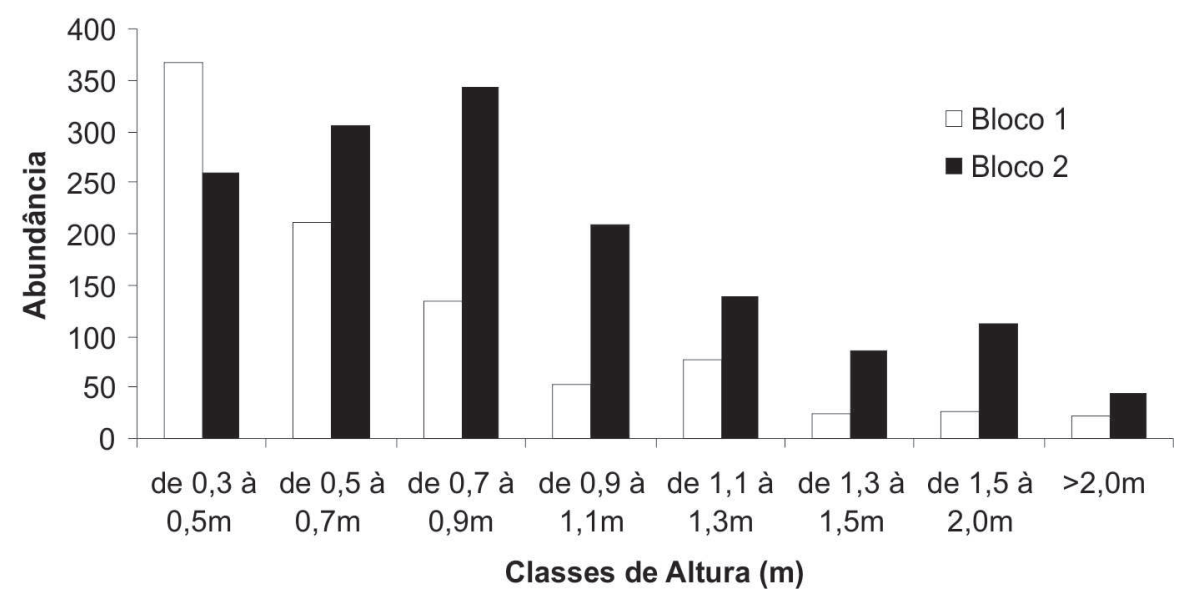

Figura 3. Distribuição das classes de altura dos indivíduos amostrados na regeneração natural sob Eucalyptus camldulensis, na Fazenda Três Irmãos, Montes Claros, MG.
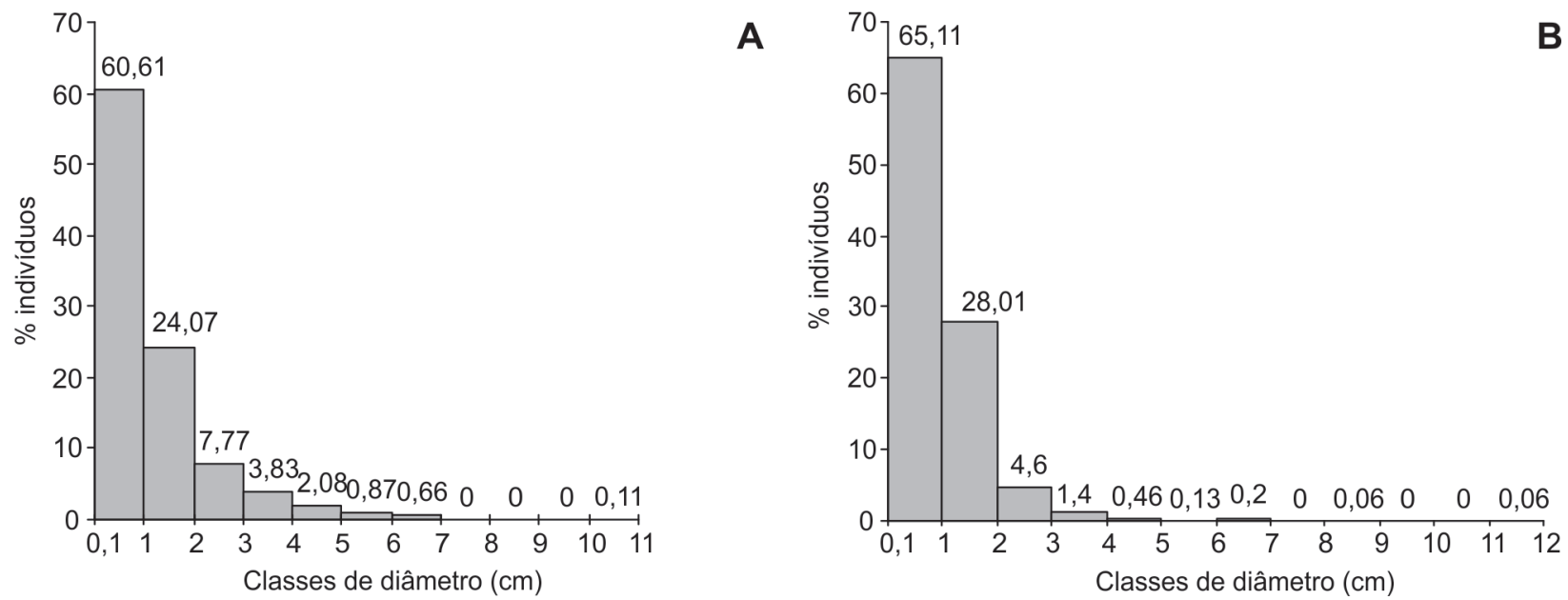

Figura 4. Distribuição das classes de diâmetro dos indivíduos amostrados na regeneração natural sob Eucalyptus camldulensis, na Fazenda Três Irmãos, Montes Claros, MG. A: Bloco 1; B: Bloco 2. 
va de Cerrado, que, possivelmente, é uma fonte de diásporos para a regeneração. O menor grau de desenvolvimento do sub-bosque neste bloco deve-se, principalmente, aos efeitos de borda e a fatores de perturbação, relativos aos processos de extração e corte do plantio de eucalipto, visto que se encontra próximo aos acessos (estradas) e, por essa razão, a atividade de máquinas e caminhões nessa área ter sido maior.

\section{CONCLUSÕES}

Os resultados apontam para a alta resiliência do Domínio Cerrado, já que, após a retirada da vegetação original, o sub-bosque formado no plantio de Eucalyptus camaldulensis apresentou alta riqueza e diversidade de famílias e espécies características do Domínio. Portanto, valoriza-se a regeneração natural como forma viável e de baixo custo para o restabelecimento da vegetação nativa.

O grau de interferência das ações antrópicas e das perturbações naturais pode ter contribuído para que a vegetação se desenvolvesse de maneira diferenciada nas áreas de borda e do interior do plantio, criando comunidades distintas, tanto estruturalmente, quanto em riqueza florística.

\section{AGRADECIMENTOS}

À FAPEMIG pela bolsa BICDT de Y. R. F. Nunes; à UNIMONTES pelo apoio logístico; à Gislene Carvalho de Castro, Laine Cristina Fernandes e Warley Ferreira da Silva pelo auxílio no trabalho de campo e a Santos D'Angelo Neto pela identificação das espécies.

\section{REFERÊNCIAS}

Alencar AL, Marangon LC, Feliciano ALP, Ferreira RLC \& Teixieira LJ (2011) Regeneração natural avançada de espécies arbóreas nativas no sub-bosque de povoamento de Eucalyptus saligna Smith., na zona da mata sul de Pernambuco. Ciência Florestal, 21:183-192.

Almeida AF (1987) Manejo ambiental nas florestas de rápido crescimento. In: III Entec - Encontro Técnico Florestal, Montes Claros, MG

APG III (2009) An update of the Angiosperm Phylogeny Group classification for the orders and families of flowering plants: APG III. Botanical Journal of the Linnean Society, 161:105121

Aubert E \& Oliveira-Filho AT (1994) Análise multivariada da estrutura fitossociológica do sub-bosque de plantios experimentais de Eucalyptus spp. e Pinus spp. em Lavras (MG). Revista Árvore, 18:215-229.

Brasil. Ministério da Agricultura. Coordenadoria de Agroenergia. (1984) Proposta de utilização energética de florestas e resíduos agrícolas. Brasília, Ministério da Agricultura. 166p.

Brower JE \& Zar, JH (1984) Field and laboratory methods for general ecology. 2.ed., Iowa, Brown Company Publishers. 226p.
Calegário N (1993) Parâmetros florísticos e fitossociológicos da regeneração natural de espécies arbóreas nativas no subosque de povoamentos de Eucalyptus, no município de Belo Oriente/ MG. Dissertação de Mestrado. Universidade Federal de Viçosa, Viçosa. 109p.

Davies-Colley RJ, Payne, GW \& Van Elswijk M (2000) Microclimate gradients across a forest edge. New Zealand Journal of Ecology, 24:111-121.

Duringan G, Franco GADC, Pastore JA \& Aguiar OT (1997) Regeneração natural da vegetação de cerrado sob floresta de Eucalyptus citriodora. Revista do Instituto Florestal, 9:71-85.

Embrapa Serviço Nacional de Levantamento e Conservação de Solos (1979) Levantamento exploratório - reconhecimento de solos do Norte de Minas Gerais área de atuação da SUDENE. Recife, Embrapa-SNLCS. 408p. Boletim Técnico, 60.

Larcher W (2000) Ecofisiologia Vegetal. São Carlos, RiMa. 531p.

Medeiros MM, Felfili JM, Libano AM (2007) Comparação florístico-estrutural dos estratos de regeneração e adulto em cerrado sensu stricto no Brasil Central. Cerne, 13:291-298.

Metzger JP \& Décamps H (1997) The structural connectivity threshold: an hypothesis in conservation biology at the landscape scale. Acta ecologica, 18:1-12.

Metzger JP (2003) Delineamento de experimentos numa perspectiva de ecologia da paisagem. In: Cullen Jr L, Rhudram R \& ValladaresPadua C (Eds.). Métodos e Técnicas na Biologia da Conservação e no Manejo da Vida Silvestre. Curitiba, UFPR. p. 539-553.

Meyer HA (1952) Struture, growth and drain in balanced unevenaged forests. Journal of Forest, 50:85-92.

Mueller-Dombois D \& Ellenberg H (1974) Aims and methods of vegetation ecology. New York, Jonh Willey \& Sons. 547 p.

Nappo ME, Fontes MAL \& Oliveira-Filho AT (2000) Regeneração natural em sub-bosque de povoamentos homogêneos de Mimosa scabrella Benth., implantadas em áreas mineradas, em Poços de Caldas - MG. Revista Árvore, 24:297-307.

Nappo ME, Fontes MAL \& Oliveira-Filho, AT (1999) Suficiência amostral e análise do tamanho de parcelas para o estudo da regeneração natural do sub-bosque de povoamentos homogêneos de Mimosa scabrella Benth., em área minerada, em Poços de Caldas-MG. Revista Árvore, 23:443-453.

Neri AV, Campos EP, Duarte TG, Meira Neto JAA, Silva AF \& Valente GE (2005) Regeneração de espécies nativas lenhosas sob plantio de Eucalyptus em área de Cerrado na Floresta Nacional de Paraopeba, MG, Brasil. Acta botanica brasilica, 19:369376.

Nunes YRF, Fagundes M, Santos RM, Domingues EBS, Almeida HS \& Gonzaga APD (2005) Atividades fenológicas em Guazuma ulmifolia Lam. (Malvaceae) em uma floresta estacional decidual no norte de Minas Gerais. Lundiana, 6:99-105.

Paciência M, Prado, J (2004) Efeitos de borda sobre a comunidade de pteridófitas na Mata Atlântica na região de Uma, sul da Bahia, Brasil. Revista Brasileira de Botânica, 27:641-653.

Pielou, EC (1975) Ecological Diversity. New York, WileyInterscience. $165 \mathrm{p}$.

Redding TE, Hope GD, Fortin MJ, Schmidt MG \& Bailey WG (2003) Spatial patterns of soil temperature and moisture across subalpine forest-clearcut edges in the southern interior of British Columbia. Canadian Journal of Soil Science, 83:121-130.

Ribeiro JF. \& Walter BMT (2008) As principais fitofisionomias do Bioma Cerrado. In: Sano SM, Almeida SP \& Ribeiro JF (Eds.) Cerrado: ecologia e flora. Planaltina, Embrapa Cerrados. p.151212 . 
Rodon Neto RM (1999) Estudo da regeneração natural e aspectos de uma clareira de formação antrópica. Dissertação de Mestrado. Universidade Federal de Lavras, Lavras. 98p.

Saporetti Jr AW, Meira Neto, JAA \& Almado R (2003) Fitossociologia de sub-bosque de cerrado em talhão de Eucalyptus grandis W. Hill ex Maiden no município de Bom DespachoMG. Revista Árvore, 27:905-910.

Sartori MS (2001) Variação da regeneração natural da vegetação arbórea no sub-bosque de Eucalyptus saligna Smith. manejado por talhadia, localizado no município de Itatinga, SP. Dissertação de Mestrado. Universidade de São Paulo, Piracicaba. 89p.

Shepherd GJ (1996) Fitopac 1: manual do usuário. Campinas, Universidade Estadual de Campinas. 96p.

Silva, E (1994) Avaliação qualitativa de impactos ambientais do reflorestamento no Brasil. Tese de Doutorado. Universidade Federal de Viçosa, Viçosa. 134p.
Sneath PH \& Sokal RR (1973) Numerical taxonomy: the principles and practice of numerical classification. San Francisco, W. H. Freeman. 553p.

Vale RS (2004) Agrossilvicultura com eucalipto como alternativa para o desenvolvimento sustentável da zona da mata de Minas Gerais. Tese de Doutorado. Universidade Federal de Viçosa, Viçosa. $101 \mathrm{p}$.

Venzke T, Neri AV, Cunha J \& Martins SV (2012) Regeneração natural do estrato arbóreo-arbustivo sob talhão de Pinus caribaea var. hondurensis, Viçosa, MG, Brasil. Global Science Technology, 5:74-86

Viani RAG, Durigan G \& Melo ACG (2010) A regeneração natural sob plantações florestais: desertos verdes ou redutos de biodiversidade?. Ciência Florestal, 20:533-552. 